Repeated physical examinations of the older employees and careful inquiry have failed to discover a symptomcomplex that could be attributed to chronic picric poisoning. If the digestive disturbances above mentioned are evidences of such poisoning, then we must admit that such a condition is very easily and rapidly acquired, as many of the men with these complaints reported shortly after being employed. The men working with this substance for the longest time show no predisposition.

This is without question a dangerous trade and many accidents occur as a natural result. Even though a company makes every effort to protect its men, trouble will still follow gross carelessness on the part of employees; and it is entirely proper to attribute a very large percentage of accidents to this cause. Also, because it is a dangerous occupation, and the men are working with something that is more or less a mystery to them, they are prone to find in their work a cause for all their bodily ills, regardless of how absurd may be the relation of cause to effect.

WOOBURN, Massachusetts

\section{A NEW DIRECT READING PRECISION REFRACTOMETER WITH UNIFORMLY DIVIDED SCALE}

By G. W. Moffit'

Received December 18, 1916

\section{I-INTRODUCTION}

It has long been known that the value of the index of refraction frequently serves as a valuable criterion of the qualities of a liquid. That the means for testing thus afforded is not in more general use seems to be due to the lack of satisfactory means for easily determining the index of refraction rather than to any inherent weakness in the method itself. If some dependable instrument combining ease of operation with accuracy of results were at hand it is probable that there are many who would avail themselves of its possibilities. While there are several good refractometers which may be obtained, their use involves a great deal of care and skill if results are to be obtained showing the necessary high degree of accuracy. In some of them the operation is long and the result is not available without more or less calculation and reference to tables.

There seems, therefore, to be a real demand in the field of food and industrial chemistry for a simple, accurate refractometer so simple in operation that one without any special knowledge of optics could obtain accurate results easily and with certainty. Such an instrument has been recently designed by the writer ${ }^{1}$ and a brief description of the refractometer and a discussion of its theory are here given for the purpose of placing the instrument before those who have need of it in their work. The good points of the instrument will appear in the discussion, and may be briefly summarized as follows:

I-A linear scale calibrated directly in terms of the index of refraction of the liquid under examination.

1 Physical Review, December, 1916.
2-The possibility of so constructing the instrument that white light may be used when it is desired to determine the mean index, the instrument still retaining approximate achromatization in many cases.

3-Simplicity, and ease of manipulation.

4-Few adjustments other than those fixed permanently in the construction of the instrument.

5 -Settings obtained by comparing the dimensions of an image with those of an eye-piece scale-an accurate means of determining the proper adjustment.

6-Adequate means for temperature control whenever required.

7--Small amount of the liquid required for a determination, a single small drop being sufficient.

8-Ability to determine the index to one or two points in the third decimal place-a degree of precision equal to, if not better, than that of the total reflection instruments.

The entire operation of determining the index of refraction of a liquid consists in placing a drop of the liquid at the proper place on a convex surface, bringing the nose of the microscope down upon it, and focusing the eye-piece. The value of the index is then read off directly from the uniformly divided scale on the focusing tube of the instrument. In an instrument of convenient size the change in setting for a change of index from 1.000 to 1.500 may be more than $10 \mathrm{~cm}$. With an eye-piece of fairly high power the uncertainty in a setting need not be more than $0.02 \mathrm{~cm}$. In most cases it will be less. The sensitiveness will, therefore, compare favorably with that of the best total reflection instruments.

\section{II-THEORY}

CASE I-Consider the arrangement shown in Fig. I. Let a point source of light be placed at $C$, distant $R$ below the surface of the liquid. Its image, due to the refraction at the surface of the liquid, will be at $S$, so that $\mathrm{ST}=\mathrm{R} / n$, where $n$ is the index of refraction of the liquid. Let $\mathrm{S}$ act as a virtual object at distance $k+\mathrm{R} / n$ from a converging lens $\mathrm{L}$ of focus $f$, and let the image of $S$ formed by this lens be at $I$, distant $p$ from the lens. Then

$$
\frac{\mathrm{I}}{k+\mathrm{R} / n}+\frac{\mathrm{I}}{p}=\frac{\mathrm{I}}{f} \text {. }
$$

Clearing of fractions,

$$
n p f+k n f+\mathrm{Rf}=p k n+\mathrm{R} p \text {. }
$$

This equation becomes linear in $n$ and $p$ when

$$
n p f=n p k \text {, or when } k=f \text {. }
$$

Equation 2 then becomes

$$
n=\frac{\mathrm{R}}{f^{2}} p-\frac{\mathrm{R}}{f} \text {. }
$$

That is, if Equation 3 is satisfied, the position of the image depends directly on the index of the liquid.

CASE 2-Consider the arrangement shown in Fig. II. Let the point source be placed at $\mathrm{C}$, the center of curvature of a spherical lens having concentric surfaces, and let a drop of the liquid be placed between the lens 


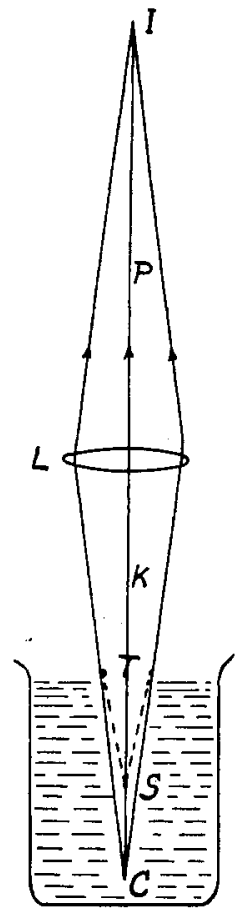

FIG.I

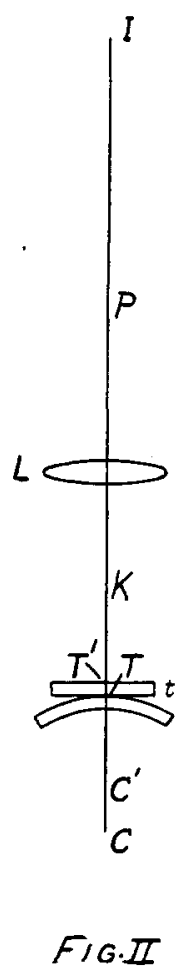

and a plane parallel glass plate placed in contact with the lens. The treatment differs from that of Case $I$ in that the effect of the plane parallel glass plate must be considered. Beginning with $\mathrm{C}$ as a point source to locate its image $C^{\prime}$ by refraction at the surface $T$ we have the following equation:

$$
\mathrm{C}^{\prime} \mathrm{T}=\frac{\mathrm{R} n^{\prime}}{n},
$$

where $n^{\prime}$ is the index of refraction of the plane parallel plate. In the same way the refraction at $T^{\prime}$ may be expressed.

$$
\mathrm{ST}^{\prime}=\left(\mathrm{C} \mathrm{T}^{\prime}+t\right) \frac{\mathrm{I}}{n^{\prime}}=\frac{\mathrm{R}}{n}+\frac{t}{n^{\prime}}
$$

where $t$ is the thickness of the plane parallel glass plate. From the equation of the converging lens,

$$
\frac{\mathrm{I}}{p}+\frac{\mathrm{I}}{\frac{\mathrm{R}}{n}+\frac{t}{n^{\prime}}+k}=\frac{\mathrm{r}}{f} .
$$

The condition that this equation be linear in $n$ and $p$ is

$$
\frac{t}{n^{\prime}}+k=f
$$

Making this substitution and clearing of fractions we have

$$
n=\frac{\mathrm{R}}{f^{2}} p-\frac{\mathrm{R}}{f} .
$$

It will be noticed that Equation 9 is identical with Equation 4, but that the conditions for linearity of scale stated in Equations 3 and 8 are slightly different, owing to the effect of the plane parallel glass plate. It will be seen that in both cases the adjustment for linearity of scale is such that if parallel rays were incident upon the upper face of the lens $L$ they would be brought to a focus at the plane surface of the liquid.

CASE 3-Consider the case when the source is not at the center of curvature of the concentric lens, or when the lens is of some other form, but at a distance from it such that its apparent distance from the curved liquid surface is $u$. The image by refraction of the liquid lens into the plane parallel plate is according to the equation for the plano-concave lens bounded on the plane side by a medium of index $n^{\prime}$.

$\frac{n^{\prime}}{\mathrm{C}^{\prime} \mathrm{T}}-\frac{\mathrm{I}}{u}=\frac{n-\mathrm{I}}{\mathrm{R}}$, or, $\mathrm{C}^{\prime} \mathrm{T}=\frac{u \mathrm{R} n^{\prime}}{\mathrm{R}+u(n-\mathrm{I})}$ (ro)

The refraction at $T^{\prime}$ may be expressed as follows:

$$
\mathrm{ST} \mathrm{T}^{\prime}=\frac{\mathrm{C}^{\prime} \mathrm{T}+t}{n^{\prime}}=\frac{u \mathrm{R}}{\mathrm{R}+u(n-\mathrm{I})}+\frac{t}{n^{\prime}}
$$

The object distance for lens $L$ is

$$
\mathrm{SO}=\frac{u \mathrm{R}}{\mathrm{R}+u(n-\mathrm{I})}+{ }_{n^{\prime}}^{t}+k .
$$

Applying the equation for the converging lens,

$$
\frac{\mathrm{I}}{p}+\frac{\mathrm{I}}{\frac{u \mathrm{R}}{\mathrm{R}+u(n-\mathrm{I})}+\frac{t}{n^{\prime}}+k}=\frac{\mathrm{I}}{f} .
$$

Differentiating with respect to $n$,

$\frac{d p}{d n}=\frac{u^{2} \mathrm{R} n^{\prime 2} f^{2}}{\left\{u \mathrm{R} n^{\prime}+\left(t+n^{\prime} k-n^{\prime} f\right)[\mathrm{R}+u(n-\mathrm{r})]\right\}^{2}}$. (I 4$)$

When the condition expressed in Equation 8 holds

$$
t+n^{\prime} k-n^{\prime} f=0
$$

and the slope is a constant, Equation $I_{3}$ taking the simpler form

$$
n=\frac{\mathrm{R}}{f^{2}} p+\left(\mathrm{I}-\frac{\mathrm{R}}{\mathfrak{u}}-\frac{\mathrm{R}}{f}\right) .
$$

When $k$ is too large the second term in the denominator is positive and increases with $n$. Therefore, the slope decreases as $n$ becomes greater and the curve is concave toward the axis of $n$. If $k$ is too small the reverse is true and the curve is concave towards the axis of $p$. If $u$ be set equal to $\mathrm{R}, \mathrm{Equation}$ I 6 becomes identical with Equation 9, that equation being a special case of the general equation just derived.

RELATIONS BETWEEN DIMENSIONS OF OBJECT AND IMAGE-Let $C$ represent the size of the object in Fig. II, and let $C^{\prime}, S$ and I represent the size of the images formed at these respective points. Then

$$
\frac{\mathrm{C}^{\prime}}{\mathrm{C}}=\frac{\mathrm{C}^{\prime} \mathrm{T}}{u}=\frac{\mathrm{S}}{\mathrm{C}}
$$

since no change in size of image is produced by the refraction at $T^{\prime}$. By refraction at the converging lens $L$,

$$
\frac{\mathrm{I}}{\mathrm{S}}=\frac{p}{\mathrm{SO}} .
$$

Combining $\mathrm{I} 7$ and $\mathrm{I} 8$,

$$
\frac{\mathrm{I}}{\mathrm{C}}=\frac{\mathrm{S}}{\mathrm{C}} \times \frac{\mathrm{I}}{\mathrm{S}}=\frac{\mathrm{C}^{\prime} \mathrm{T}}{u} \times \frac{p}{\mathrm{SO}} .
$$

Substituting the values of $C^{\prime} T, p$, and SO from Equa-tions $10, I 2$ and $I 6$, and simplifying, 


$$
\mathrm{I}=\frac{f}{\mathrm{C}} \text {. }
$$

The image is of constant size for a fixed value of $u$, a property of the system utilized in a method for precise focusing to be described in a later paragraph.

$$
\text { III-ABERRATIONS }
$$

SPHERICAL ABERRATION AND DISTORTION-With the direct illumination that may be used in this instrument it is possible to use a stop of rather small diameter and still have a well-lighted field of view. Since only a small portion of each lens is used in forming the image of any point in the object the effect of spherical aberration in blurring the image is very small. Therefore, it seems unnecessary to take particular precautions to eliminate spherical aberration.

Curvature of the image, however, may affect the performance of the instrument to a slight extent. The curvature of the virtual image formed by the liquid lens is concave towards the eye-piece. The effect of the converging lens $L$ in forming the image at the eyepiece is to diminish the curvature. The curvatures of the surfaces and the position of the stop should be so chosen that the resulting image will be as nearly plane as possible over the area covered by the image at the eye-piece.

Even if the radius of curvature of the image were so small as $5 \mathrm{~cm}$. in an instrument whose image were 0.5 $\mathrm{cm}$. in extent, the periphery of the image would be out of the plane of the center by

$$
h=\frac{d^{2}}{8 \mathrm{R}}=\frac{25}{400}=0.06 \mathrm{~mm} \text {. }
$$

This value is less than the error of a setting. And since the focusing is always done on the peripheral portions of the image the effect would be to change slightly the position of the zero point of the scale.

CHROMATIC ABERRATION-In considering the chromatic properties of the system two distinct cases arise. If the converging lens $\mathrm{L}$ be strictly achromatic the adjustment for $k$ is correct for all wave-lengths. The instrument will not give an achromatic image at the eye-piece, but the various colored images will be distributed along the axis according to the dispersion of the particular liquid in the apparatus. Such would be the construction of the refractometer if it is desired to measure the value of the index for different wavelengths in order to determine the dispersion of the liquid. In this case monochromatic illumination is, of course, necessary. Lens $L$ must be thoroughly achromatized in order that the adjustment of $k$ shall be correct for all the values of wave-length with which the instrument is to be used.

The other case arises when the lens $L$ is of the simple uncorrected type. This leads to a construction which may be approximately achromatized for liquids of ordinary dispersion, and which when illuminated with white light will give the index for some particular value of the wave-length for which the instrument has been calculated. Considering the positions of the red and the blue images formed by the liquid alone, we find that the blue image lies nearer the lens $L$ than does the red image. If the chromatic properties of this lens are such that its images of the images formed by the liquid lens fall at the same point the system will be achromatic in the sense that the different colored images will be at the same point in the eyepiece. This condition can be realized only for liquids whose dispersion bears a certain relation to the index of refraction for some intermediate wave-length. Since no such relation is general it will be seen that it is not possible to achromatize the instrument completely, although an approximation may be realized which will be satisfactory for many liquids. Whenever it is desired to use the instrument with a liquid which gives chromatic effects detrimental to accurate focusing it would be necessary to use the monochromatic light for which the instrument was designed to give accurate readings.

The condition for approximate achromatization may be derived as follows: For the convergent lens L, assumed bi-convex,

$\frac{\mathrm{I}}{\mathrm{V}^{\prime} \mathrm{F}}-\frac{\mathrm{I}}{\mathrm{V}^{\prime} \mathrm{C}}=\frac{\mathrm{V}^{\prime} \mathrm{C}-\mathrm{V}_{\mathrm{F}}^{\prime}}{\mathrm{V}^{\prime}{ }_{\mathrm{C}} \mathrm{V}^{\prime} \mathrm{F}}=\frac{\mathrm{V}^{\prime} \mathrm{C}-\mathrm{V}^{\prime} \mathrm{F}}{\mathrm{V}^{\prime 2} \mathrm{D}}=\frac{2 d^{\prime}}{r}$,

where $V^{\prime} F$ and $V^{\prime} C$ are the virtual object distances for the lens $L$ for the same value of $p$, and where $d^{\prime}=n^{\prime} F-n_{C}^{\prime}$ for the lens, and $r$ is the radius of curvature of the lens faces. For the plano-concave liquid lens,

$$
\frac{\mathrm{V}_{\mathrm{C}}-\mathrm{V}_{\mathrm{F}}}{\mathrm{V}_{\mathrm{F}} \mathrm{V}_{\mathrm{C}}}=\frac{\mathrm{V}_{\mathrm{C}}-\mathrm{V}_{\mathrm{F}}}{\mathrm{V}_{\mathrm{D}}{ }^{2}}=\frac{d}{\mathrm{R}^{\prime}}
$$

where $V_{C}$ and $V_{F}$ are the distances from the liquid to the corresponding images for a given value of $u$. $\mathrm{R}$ is the radius of curvature for the curved surface of the liquid and $d=n_{\mathrm{F}}-n_{\mathrm{C}}$. The condition for achromatization is

$$
\mathrm{V}_{\mathrm{C}}-\mathrm{V}_{\mathrm{F}}=\mathrm{V}_{\mathrm{C}} \mathrm{C}-\mathrm{V}_{\mathrm{F}}^{\prime}
$$

Dividing (21) by (22) and substituting (23),

$$
\frac{\mathrm{V}_{\mathrm{D}}^{2}}{\mathrm{~V}^{\prime} \mathrm{D}^{2}}=\frac{2 \mathrm{R} d^{\prime}}{r d}
$$

This reduces to

$$
\begin{aligned}
& \left.u \mathrm{R} \sqrt{2 f_{\mathrm{D}}\left(n^{\prime} \mathrm{D}\right.}-\mathrm{I}\right) d= \\
& n_{\mathrm{D}} f_{\mathrm{D}} u \sqrt{2 \mathrm{R} d^{\prime}}+\left(u \mathrm{R}+f_{\mathrm{D}} \mathrm{R}-f_{\mathrm{D}} u\right) \sqrt{2} \mathrm{R}^{\prime},
\end{aligned}
$$

which shows that it is possible to achromatize the system for any liquid whose mean index bears a certain relation to the square root of its dispersion. This relation is determined by the constants of the optical system.

$$
\text { IV-THE INSTRUMENT }
$$

The refractometer may be set up in a convenient form resembling an ordinary microscope in appearance. Instead of the usual state a heating tank may be provided, through the center of which passes a springmounted tube carrying the object scale at the lower end and the lens on which the liquid is placed at the upper end. The spring mounting lifts this tube so that the lens comes above the top of the heating jacket when the microscope is raised. In this position it is easy of access for cleaning and for placing the drop 
of liquid in position. At the lower end of the microscope tube is placed the plane parallel plate, and up in the tube at the proper distance is placed the converging lens $I$. The microscope tube is arranged so that it may belowered into position and the two lenses brought into contact, and in contact with the liquid, and lowered into the heating jacket. The eye-piece should be mounted in the end of a tube telescoping into the microscope tube with rack and pinion adjustment for ease of focusing. The focusing tube should also be provided with a scale, read with a vernier, whose divisions are in accord with Equation $I 6$, if the instrument is to be direct reading. The eye-piece should be of fairly high power and should be mounted in such manner that it and the eye-piece scale may be moved laterally a short distance in order to enable the user to bring the image and the scale into coincidence.

ADJUSTMENTS-The adjustments of the instrument are few and simple. The converging lens and the plane parallel plate must be carefully fixed with respect to each other. On this adjustment depends the linearity of scale. It will be seen that the proper adjustment is such that parallel light incident on the lens from above is brought to a focus on the lower surface of the plate. If the system be lined up with a telescope focused for parallel light by the auto-collimation method, the adjustment may be made by eliminating parallax between the cross wire of the telescope and the images of fine dust particles on the lower surface of the plate. If the lens is not achromatic this adjustment should be made with monochromatic light of the same wave-length for which the instrument is to read correctly.

FocUSING-In the development of the equations it was shown that the image in the eye-piece is of a fixed size whenever the instrument is in proper focus. The ratio of the image size to the object size is given in Equation 20. Therefore, if two scales be ruled with divisions in the ratio of $f$ to $u$ the one may be used as an eye-piece scale and the other as an object whose image is formed at the eye-piece. When focusing is complete, image and eye-piece scale will be of the same size and coincident.

POSITION OF STOPS: EFFECT ON FOCUSING-The rays usually used when locating the position of an image graphically are seldom those that actually form the image. The position of the stop that limits the aperture of the instrument determines the rays that actually form each part of the image. This would seem to be of small importance here in its effect on spherical aberration and curvature of field but it plays an important part in focusing by comparison of image with eye-piece scale. While the eye-piece is moved outward throughout the range of clear focus we may have the image increasing, decreasing, or remaining of constant size, depending on the position of the stop. And the steeper the slope of the chief image rays with the axis of the instrument the greater will be the accuracy with which the focusing may be done. Several cases arise.
I-When the stop is between the object and the liquid lens the chief image rays are slightly convergent and the image increases in size as the eye-piece is racked in. This convergence is never very great. A rather large aperture for the liquid lens is also required. For these reasons this position of the stop is not good.

2- When the stop is in the plane of the liquid lens it is at the principal focus of the lens $L$ and the chief image rays are parallel. No change in the size of the image restults on moving the eye-piece toward or away from the lens. Accurate focusing cannot be done with the stop in this position.

3 -When the stop is at the converging lens the chief image rays diverge from a point approximately coincident with the optical center of the lens. Their slope decreases with increase of the index of the liquid, thus decreasing the accuracy of a setting for the higher

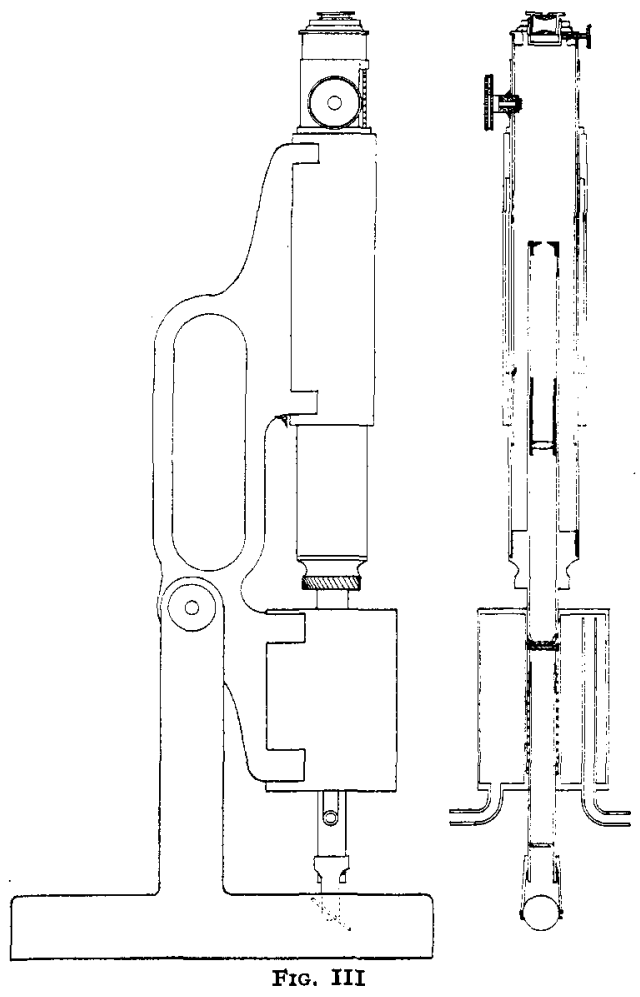

values of the index. It is apparent, however, that the decrease in accuracy is less rapid than the increase in value of the index so that the percentage error decreases with increase of the index. It will be noted that the center of the liquid lens is used to form the center of the image while the peripheral rays form the edge of the image. This arrangement enables one to focus with a good degree of precision.

4-If the stop be placed at a fixed distance above the converging lens, say at its principal focus, the divergence of the chief image rays is increased over that of Case 3 , and the accuracy of a setting thereby increased. Between the liquid lens and the converging lens the chief rays are parallel to the principal axis. The effective apertures of both lenses are the same and vary inversely with the index of the liquid. The percentage accuracy of the settings is a constant because the distance from the stop to the image is directly propor- 
tional to the index of the liquid. This position of the stop seems most likely to give high precision in the readings. Evidently the most accurate settings can be made when the divergence of the chief image rays is as great as is consistent with a good length of image and reasonably small apertures of the lenses, especially of the liquid lens. Since the aperture of the lenses is increased by bringing the stop nearer the eye-piece it is seen that there is a limit where the increase in aberration effects due to increased aperture of the lenses will counterbalance any gain in accuracy of focusing due to increased divergence of the chief image rays.

A compact instrument with a very open scale, and covering a long range, might be constructed in the following manner: Let the spring-mounted tube carry three object scales instead of one. Let these scales be so ruled and placed that when focusing on the first scale values of index from I.000 to I. $2+$ would cover the range of the engraved scale of the instrument. By focusing on the second scale values from I.2 to I. 4 + could be read, and by focusing on the third scale values from I.4 to $I .6+$ could be determined. Such an instrument would be of practically the same size as one with single scale covering the same range with one-third the openness of scale. With certain values of index two scales would be in focus in the field of view at the same time. Each scale should be given a distinguishing mark and the three should be placed at different angles across the field of view in order to prevent confusion of the scales.

\section{V-EXPERIMENTAL}

After considerable preliminary work a single scale instrument was constructed essentially as described above. The lenses, except the optical flat, were made by a local firm of spectacle lens grinders. The centering was not all that could be desired and for this reason a satisfactory study of the effect of varying the position of the stop was impossible. The stop was placed at the converging lens $L$ which had a focal length of about $8.5 \mathrm{~cm}$. The aperture used was somewhat less than a centimeter in diameter. The radius of curvature of the upper face of the concentric lens was $6.0+\mathrm{cm}$. Actual openness of scale was found to be $1.282 \mathrm{~cm}$. for a change of $0 . I$ in the index of the liquid. The value of $u$ was placed equal to that of $f$, since that adjustment leads to the shortest tube length for the instrument. It also leads to more accurate focusing than when the tube is longer since the chief image rays are more divergent. The length of the object and the image scales was $0.5 \mathrm{~cm}$. The approximate adjustment of $k$ was made by the collimation method already described, using sodium light.

Careful determinations of the index of refraction were then made for several liquids using a spectrometer and a hollow $60^{\circ}$ prism. Immediately after the deviation readings were taken with the spectrometer a drop of the liquid was removed from the prism and placed in the refractometer. A number of settings were made and the reading of the centimeter scale on the focusing tube of the instrument recorded each time. The illumination for all readings was furnished by a Bunsen burner whose flame was colored by a piece of asbestos soaked in a solution of common salt. The procedure was carried out for all the liquids in the table below.

\begin{tabular}{|c|c|}
\hline Subs & $\begin{array}{l}\text { Spectrometer } \\
\text { Value of } n\end{array}$ \\
\hline 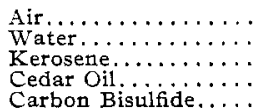 & $\begin{array}{l}1.000 \text { (assumed) } \\
1.3318 \\
1.4475 \\
1.4994 \\
1.6209\end{array}$ \\
\hline
\end{tabular}

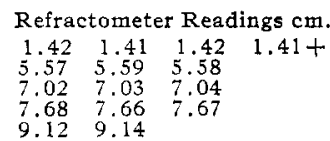

Curve I, Fig. IV, shows the results graphically. It will be noticed that the curve deviates somewhat from a straight line, being concave toward the axis of index of refraction. If we refer to Equation I4, which gives the slope of this graph as $n$ varies, we see

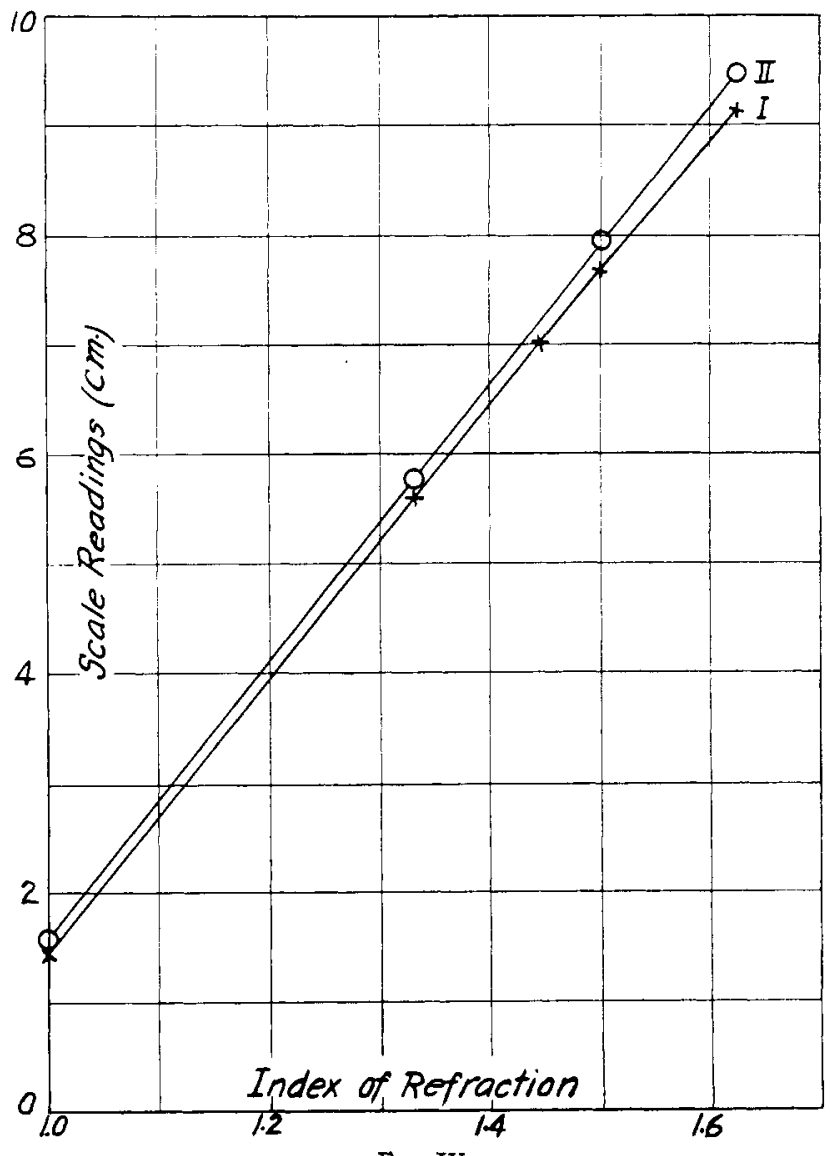

Fig. IV

that the curve would be concave toward the axis of indices when the value of $k$ is too great. The value of $k$ was then decreased by $0.03 \mathrm{~cm}$. and readings again taken. The results are shown in Curve II, Fig. IV. The graph is sensibly a straight line.

It is obvious that if a uniformly divided scale be placed so that the 1.000 mark shall coincide with the $1.55 \mathrm{~cm}$. mark of the centimeter scale on this instrument as used in making the above readings, and the 1.600 mark be made to coincide with the $9.23 \mathrm{~cm}$. mark we shall have a direct reading instrument with an openness of scale sufficient to determine the index to the third decimal place.

This refractometer has been made the subject of patent application, and its manufacture will be taken up if sufficient demand arises.

WASHINGTON UNIVERSITY ST. LouIs, MIssourI 